\title{
An Eggshell-Based Toothpaste as a Cost-Effective Treatment of Dentin Hypersensitivity
}

\author{
Ensanya A. Abou Neel ${ }^{1,2,3}$ Turki A. Bakhsh ${ }^{4}$ \\ ${ }^{1}$ Department of Preventive and Restorative Dentistry, College \\ of Dental Medicine, University of Sharjah, Sharjah, United Arab \\ Emirates \\ 2Department of Biomaterials, Faculty of Dentistry, Tanta University, \\ Tanta, Egypt \\ ${ }^{3}$ Division of Biomaterials and Tissue Engineering, UCL Eastman \\ Dental Institute, Royal Free Hospital, UCL Medical School, \\ Hampstead, London, United Kingdom \\ ${ }^{4}$ Department of Restorative Dentistry, Faculty of Dentistry, King \\ Abdulaziz University, Jeddah, Saudi Arabia
}

Eur J Dent 2021;15:733-740.

\begin{abstract}
Address for correspondence Ensanya A. Abou Neel, PhD, Department of Preventive and Restorative Dentistry, College of Dental Medicine, University of Sharjah, P.O. Box, 27272, Sharjah, United Arab Emirates (e-mail: eabouneel@sharjah.ac.ae; e.abouneel@ucl.ac.uk).
\end{abstract}

\begin{abstract}
Keywords

- eggshell

- titanium dioxide nanoparticles

- dentin hypersensitivity

- optical coherence tomography

- scanning electron microscopy
\end{abstract}

Objectives This study aimed to test the efficacy of two experimentally produced eggshell-based toothpastes on dentinal tubules occlusion.

Materials and Methods Two experimental eggshell toothpastes, based on natural ingredients, with or without titanium dioxide nanoparticles (TNPs) were produced and coded "TNPs eggshell toothpaste [TNPsESTP]" or "eggshell toothpaste [ESTP]," respectively. Mid-coronal dentin discs, from 28 human extracted molar teeth, etched with $37 \%$ phosphoric acid for 60 seconds to simulate the hypersensitive dentin, were randomly divided into four groups-G1: no treatment (negative control), G2: ESTP treated, G3: TNPsESTP treated, and G4: Biorepair treated (positive control). All treated discs were brushed for 2 weeks and 1 month using a toothbrush simulator at $40 \mathrm{~mm} / \mathrm{s}$. Dentinal tubules occlusion was studied using a cross-polarization optical coherence tomography (CP-OCT) and scanning electron microscopy (SEM).

Statistical Analysis Unpaired $t$-test was conducted using GraphPad software (San Diego, California, United States). Continuous variables were expressed as means \pm standard deviation and $p<0.05$ was considered statistically significant.

Results From CP-OCT, a marked increase in surface reflectivity of dentin was observed after brushing with tested toothpastes. ESTP and NPsESTP showed higher or comparable grayscale values than Biorepair indicating increase in surface density of dentin. From SEM, at 2 weeks, ESTP showed comparable number of completely occluded dentinal tubules to Biorepair. TNPsESTP showed significantly lower numbers of CODT than Biorepair. At 1 month, the number of CODT was difficult to count for all treated groups. Both ESTP and TNPsESTP showed significantly higher numbers of partially occluded dentinal tubules than Biorepair.

Conclusion ESTP or TNPsESTP could offer a cost-effective substitute for the treatment of dentin hypersensitivity. published online

August 24, 2021
DOI https://doi.org/

10.1055/s-0041-1729676 ISSN 1305-7456
(C) 2021. European Journal of Dentistry.

This is an open access article published by Thieme under the terms of the Creative Commons Attribution-NonDerivative-NonCommercial-License, permitting copying and reproduction so long as the original work is given appropriate credit. Contents may not be used for commercial purposes, or adapted, remixed, transformed or built upon. (https://creativecommons.org/licenses/by-nc-nd/4.0/).

Thieme Medical and Scientific Publishers Pvt. Ltd. A-12, 2nd Floor, Sector 2, Noida-201301 UP, India 


\section{Introduction}

Dentin hypersensitivity (DH) is a major health care problem affecting both younger and older populations. It is characterized by a short, sharp pain of unexplained reason that might occur in response to chemical (e.g., excessive acidic drinks or bleaching) or mechanical factors (e.g., vigorous tooth brushing). In older population, DH can be associated with root exposure especially after periodontal treatment. ${ }^{1}$

The most accepted theory, explaining the pain associated with $\mathrm{DH}$, is "hydrodynamic" that relates it to the stimulation of baroreceptors in response to fluid movement within dentinal tubules. The more opened the dentinal tubules, the more sever the pain. Occlusion of dentinal tubules was therefore considered as the main treatment option for reducing $\mathrm{DH}^{2-4}$ Many commercially available toothpastes have been proposed for such purpose. They depend on the incorporation of active ingredients with remineralizing potential $^{5}$ such as nanohydroxyapatite (e.g., Biorepair Fast Sensitive Repair), ${ }^{6}$ casein phosphopeptide-amorphous calcium phosphate (e.g., GC tooth mousse), ${ }^{7}$ or fluoride (e.g., Sensodyne Repair \& Protect). ${ }^{8}$ However, there is no gold standard toothpaste that could afford complete and persistent cure. The action of most toothpastes is only temporary and any dietary challenge (e.g., acidic drinks) might remove the blocking agent and reopen the dentinal tubules. Due to the slow release of some active ingredients, the effectiveness of these toothpastes might take several weeks.

Recently, the use of natural products as a cost-effective treatment option has gained popularity. Eggshell powder (ESP) has attracted researchers' attention as enriched vitamin-mineral supplements for postmenopausal women for the prevention and treatment of osteoporosis. ${ }^{9,10} \mathrm{ESP}$ is a rich source of calcium, phosphorus, strontium, zinc, fluoride, and copper that could help in remineralization. ${ }^{11,12}$ It increases bone density, ${ }^{13}$ and reduces resorption and pain. When tested in rats and human, it shows antirachitic effect. ${ }^{14}$ In dentistry, ESP found an interest as a pulp capping material ${ }^{15}$ and in treating enamel erosion. ${ }^{16}$

This study is therefore aimed to develop toothpastes based on: (1) ESP as a rich source of ions for remineralization, (2) potato powder as a source of fluoride, ${ }^{17}$ (3) baking soda as a source of sodium, (4) titanium dioxide nanoparticles (TNPs) due to its antibacterial action, ${ }^{18}$ and (5) clove oil due to its sedative $^{19}$ and inhibitory action on demineralization. ${ }^{20}$ The efficacy of these experimental toothpastes on dentin remineralization was compared with "Biorepair Fast Sensitive Repair." The null hypothesis was "there is no significance in the difference between experimental toothpastes and Biorepair Fast Sensitive Repair on dentinal tubules' occlusion."

\section{Materials and Methods}

\section{Experimental Toothpaste Preparation}

The composition of the two experimental toothpastes as well as Biorepair Fast Sensitive Repair that was used as a positive control is given in - Table 1 .

\section{Preparation of Dentin Discs}

A total of 28 human extracted third molar teeth were used after getting ethical approval (no. 102-11-17). Mid-coronal dentin discs of approximately $1 \mathrm{~mm}$ thickness were prepared using a diamond saw (TechCut 4TM, Allied, United States) and mounted in a self-cure acrylic resin. The discs were smoothed with \#240, 400, and 600 grit silicon carbide, etched with 37\% phosphoric acid for 60 seconds, ultrasonically washed with distilled water for 2 minutes and left to air dry for few minutes. Etched dentin discs were then randomly divided into four groups as follow: G1: etched dentin received no treatment as a negative control $(n=4)$, G2: etched dentin brushed with eggshell toothpaste (ESTP) ( $n=8)$, G3: etched dentin brushed with TNPs eggshell toothpaste (TNPsESTP) $(n=8)$, and G4: etched dentin brushed with Biorepair as a positive control $(n=8)$.

\section{Tooth Brushing Simulation}

A slurry from each toothpaste was prepared at a toothpaste: distilled water ratio of $1: 3$. Samples were mounted on the toothbrush simulator (ZM-3.8, SD Mechatronic GmbH, Germany) and approximately $10 \mathrm{~mL}$ of the toothpaste slurry was used to cover each dentin disc. The tooth brushing was carried out using classic soft brushes at $40 \mathrm{~mm} / \mathrm{s}$ in a circular motion to simulate a period of 2 and 4 weeks. Assuming that the brushing is three times/day; each time takes 3 minutes and the number of teeth is 28 , the brushing time (minute) for each tooth, that corresponds to 2 or 4 weeks, was calculated from the following equation:

Brushing time minute $=\quad$ No. of brushing per day $\times$ time of brushing (minute) No. of teeth in a mouth $\times$ no. of days

The brushing time was then converted into cycles, taking into account that each cycle takes a second (i.e., 60 cycles/min) using the following equation:

No. of brushing cylces $=$ Brushing time minute $\times$ no. of cycles per minute

At each time point, dentin discs were removed and washed with distilled water and left to air dry.

Table 1 Composition of experimental and control toothpastes used in this study

\begin{tabular}{|l|l|}
\hline $\begin{array}{l}\text { Toothpaste } \\
\text { (code) }\end{array}$ & Composition \\
\hline $\begin{array}{l}\text { Eggshell tooth- } \\
\text { paste (ESTP) }\end{array}$ & $\begin{array}{l}7 \mathrm{~g} \text { eggshell powder } \\
1 \mathrm{~g} \text { potato powder } \\
1 \mathrm{~g} \text { baking soda } \\
1 \mathrm{~g} \text { oil of clove }\end{array}$ \\
\hline $\begin{array}{l}\text { Titanium dioxide } \\
\text { nanoparti- } \\
\text { cles-containing } \\
\text { eggshell tooth- } \\
\text { paste (TNPs-ESTP) }\end{array}$ & $\begin{array}{l}1 \mathrm{~g} \text { gaking powder } \mathrm{g} \text { titanium dioxide nanoparticles } \\
1 \mathrm{~g} \text { oil of clove }\end{array}$ \\
\hline $\begin{array}{l}\text { Biorepair Fast } \\
\text { Sensitive Repair } \\
\text { (Biorepair) }\end{array}$ & $\begin{array}{l}\text { Aqua, zinc hydroxyapatite, glycerin, sorbitol, } \\
\text { silica, PEG-32, cellulose gum, aroma, } \\
\text { sodium myristoyl sarcosinate, sodium } \\
\text { methyl cocoyl taurate, citric acid, phenox- } \\
\text { yethanol, benzyl alcohol, sodium benzoate }\end{array}$ \\
\hline
\end{tabular}




\section{Cross-Polarization Optical Coherence Tomography}

A cross-polarization optical coherence tomography (CP-OCT; IVS-300, Santec, Japan) was used to examine the dentin samples. A continuous wavelength scanning diode laser centerd near $1,310 \mathrm{~nm}$ with a wavelength range of $100 \mathrm{~nm}$ was used at a scanning rate of $30 \mathrm{kHz}$, axial resolution of approximately $12 \mu \mathrm{m}$ and lateral resolution of $30 \mu \mathrm{m}$. The samples were scanned in a noncontact mode. The size of the cross-section (B-scan; two-dimensional [2D]) was about $500 \times 924$ pixels which correspond to $5 \times 8.18 \mathrm{~mm}^{2}(X, Z)$. The specimen was placed on a micrometer stage with the treated surface positioned perpendicular to the projected OCT light source and away from the handheld OCT probe by $0.5 \mathrm{~mm}$ to obtain three tomographic images at every $500 \mu \mathrm{m}$. The obtained OCT data were analyzed using an image analysis software (ImageJ v. 1.45q, National Institutes of Health).21,22 The obtained data were converted to eight-bit grayscale, followed by adjusting the dentin surface with horizontal plane (zero degree) followed by adjusting the scale according to the acquired OCT data (pixel size). Then, five equal areas $(0.20 \mathrm{~mm}$ width $\times 0.50 \mathrm{~mm}$ depth $)$ as a representative dimension of the area of interest (AOI) were selected within the outer dentin surface at different locations. Then, the maximum pixel values (MPVs) of AOI were measured from the OCT grayscale image by analyzing the intensity of light by linearly scaling the pixels from minimum to maximum, which is basically 0 to 255 as a display range where zero corresponds to black pixel (i.e., low signal interference), while 255 corresponds to white pixel (i.e., high signal scattering and interference).

\section{Scanning Electron Microscopy}

Dentin discs were dried using serial dilution of alcohol, critically dried for 30 minutes, mounted on metal stubs, coated with carbon, and examined using scanning electron microscopy (SEM, JEOL JSM6480LV, United States) after sputter coating. Mean \pm standard deviation (SD) of the diameter and number of opened, partially closed, and completely closed dentinal tubules in an area of $125 \times 90 \mu^{2}$ for each group after 2 weeks and 1 month was calculated.

\section{Statistical Analysis}

For the number of opened and partially closed dentinal tubules, the unpaired $t$-test was conducted using GraphPad software (San Diego, California, United States). Continuous variables were expressed as means \pm SD and $p<0.05$ was considered statistically significant.

\section{Results}

\section{Cross-Polarization Optical Coherence Tomography}

In OCT imaging, the detected interference along the course of the laser beam at a specific location inside a scattering biological structure is described as A-scan, which demonstrated as a profile of signal intensity. A compilation of A-scan with different signal intensity values will reconstruct a 2D cross-sectional grayscale image of the scanned structure (B-scan). After processing the raw data, B-scans showed areas with different gray shades. Some areas were showing high signal intensity in the form of bright pixels, while other areas were showing low signal intensities in the form of dark pixels. The representative B-scan, surface topography, and surface reflectivity of negative control ( $\neg$ Fig. 1), ESTP ( - Fig. 2), TNPsESTP ( - Fig. 3 ) and Biorepair positive control ( - Fig. 4 [I and II]) groups showed a marked increase in surface reflectivity of dentin with increasing time of paste application. The collective MPV value for all groups at 2 weeks and 1 month is shown in - Fig. 4 (III). As seen, at 2 weeks, the MPV follows this order: TNPSESTP $(191)>$ ESTP $(187)>$ negative control (174) > Biorepair (170). At 1 month, the MPV follows this order: ESTP (221) > TNPsESTP $=$ Biorepair $(204)>$ negative control (174). The MPV for all treated groups was higher at 1 month compared with 2 weeks.

\section{Scanning Electron Microscopy}

-Fig. 5 shows the SEM images of etched dentin. As seen all dentinal tubules were patent with an average diameter of 2.8 $\pm 0.9 \mu \mathrm{m}$. After brushing with the ESTP, the occlusion of dentinal tubules was observed. As a result, a reduction in diameter of dentinal tubules occurred ( $\boldsymbol{-}$ Table 2 ). For all treated groups, a complete occlusion of dentinal tubules started at 2 weeks and increased at 1 month. Therefore, it was difficult to count the number of completely occluded tubules at 1 month. The number of completely closed dentinal tubules in an area of $125 \times 90 \mu \mathrm{m}^{2}$ followed this order Biorepair > ESTP > TNPsESTP. At 2 weeks, only TNPsESTP showed significantly lower number than Biorepair. A reverse order was observed regarding the number of opened or partially closed dentinal tubules where TNPsESTP > ESTP > Biorepair.
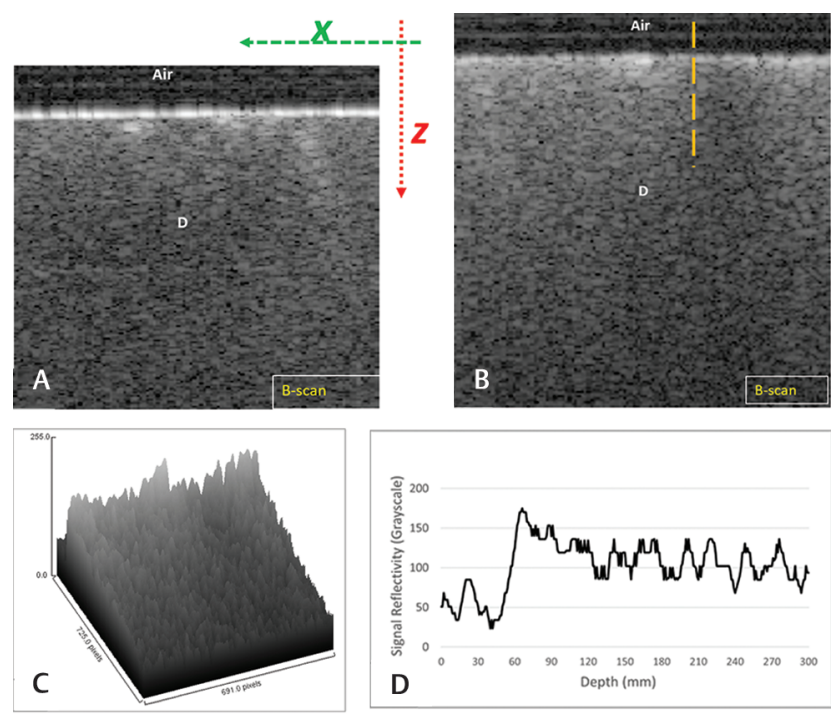

Fig. 1 Etched dentin (negative control) group: Representative B-scan shows the gradual decrease in the signal intensity on Z-axis (A). Typical OCT image (B) and its analysis (C,D) showed the presence of a band of bright pixels along the surface; the broken line representing A-scan. The surface plot (C) showed the presence of several high peaks of bright pixels at the dentin surface with gradual decrease at deeper level. The plot profile of A-scan (D) shows the MPV at outer surface. D, dentin; MPV, maximum pixel value; OCT, optical coherence tomography. 

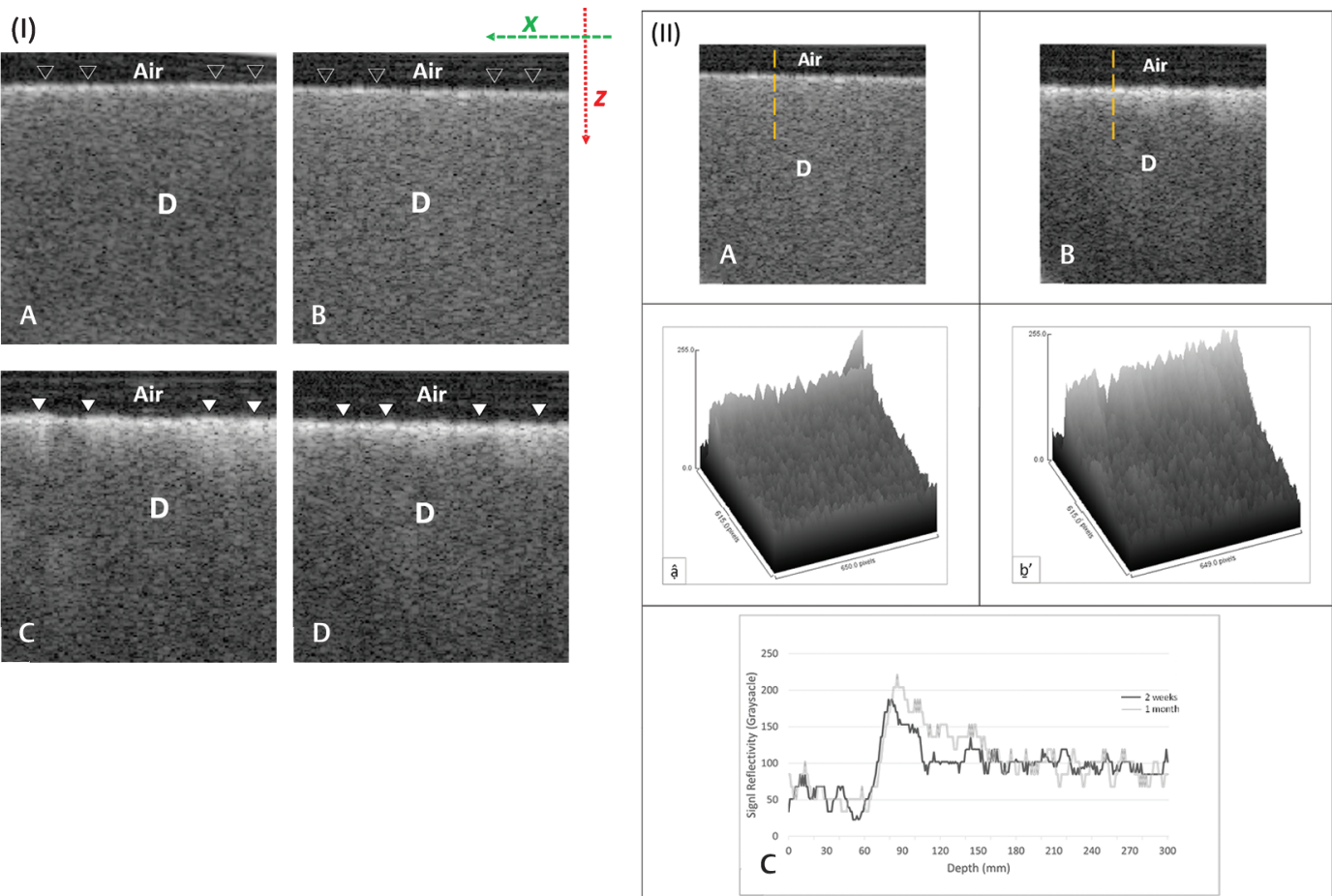

Fig. 2 ESTP group: (I) Representative B-scans after 2 weeks (A, B) and 1 month (C, D). Constant application of ESTP for 1 month has markedly increased the surface reflectivity of the dentin as indicated by the solid white triangles in comparison to the same area indicated by hollow triangles. (II) Representative OCT scans and their signals analyses after 2 weeks (a, ậ) and 1 month (b, b'); the vertical broken lines represent A-scan. As seen, an intense backscattered reflection was observed after 1 month (b) in comparison to the light bright band of pixels seen after 2 weeks (A). The presented surface plots (ậ, b'), correspond to the OCT scans in (a) and (b), respectively, showed modifications in the surface density of dentin as indicated by the variation in grayscale peaks. The signal reflectivity at the outer dentin surface after 1 month was greater than 2 weeks (c). D, dentin; ESTP, eggshell toothpaste; OCT, optical coherence tomography.

Table 2 Mean \pm SD of the diameter and number of dentinal tubules in an area of $125 \times 90 \mu \mathrm{m} 2$ for each group after 2 weeks and 1 month

\begin{tabular}{|c|c|c|c|c|c|c|}
\hline \multirow[t]{2}{*}{ Sample } & \multicolumn{6}{|c|}{ Diameter of dentinal tubules $(\mu \mathrm{m})$} \\
\hline & \multicolumn{3}{|l|}{$2 \mathrm{wk}\left({ }^{*}\right) /(¥)$} & \multicolumn{3}{|l|}{$1 \mathrm{mo}\left({ }^{*}\right) /(¥)$} \\
\hline ED & \multicolumn{6}{|c|}{$2.8 \pm 0.9$} \\
\hline ESTP & \multicolumn{3}{|c|}{$1.3 \pm 0.4(0.02) /(0.16)$} & \multicolumn{3}{|c|}{$1.7 \pm 0.5(0.07) /(0.03)$} \\
\hline TNPsESTP & \multicolumn{3}{|c|}{$1.5 \pm 0.3(0.03) /(0.03)$} & \multicolumn{3}{|c|}{$1.5 \pm 1.0(0.1) /(0.24)$} \\
\hline Biorepaire & \multicolumn{3}{|c|}{$0.9 \pm 0.3(0.007)$} & \multicolumn{3}{|c|}{$0.8 \pm 0.4(0.006)$} \\
\hline \multicolumn{7}{|c|}{ Number of dentinal tubules in area of $125 \times 90 \mu \mathrm{m}^{2}$} \\
\hline \multirow[t]{2}{*}{ Sample } & \multicolumn{3}{|l|}{$2 \mathrm{wk}$} & \multicolumn{3}{|l|}{$1 \mathrm{mo}$} \\
\hline & $\begin{array}{l}\text { ODT } \\
\left({ }^{*}\right) /(¥)\end{array}$ & $\begin{array}{l}\text { PCDT } \\
\left({ }^{*}\right) /(¥)\end{array}$ & $\begin{array}{l}\text { CCDT } \\
\left({ }^{*}\right) /(¥)\end{array}$ & $\begin{array}{l}\text { ODT } \\
\left({ }^{*}\right) /(¥)\end{array}$ & $\begin{array}{l}\text { PCDT } \\
\left({ }^{*}\right) /(¥)\end{array}$ & $\begin{array}{l}\text { CCDT } \\
\left({ }^{*}\right) /(¥)\end{array}$ \\
\hline ED & $198 \pm 2$ & - & - & $198 \pm 2$ & - & - \\
\hline ESTP & $43 \pm 1(0.0001) /(0.001)$ & $\begin{array}{l}90 \pm 15 / \\
(0.38)\end{array}$ & $\begin{array}{l}45 \pm 11 / \\
(0.18)\end{array}$ & $4 \pm 1(0.0001) /(0.20)$ & $\begin{array}{l}105 \pm 15 / \\
(0.009)\end{array}$ & a \\
\hline TNPsESTP & $\begin{array}{l}106 \pm 6(0.0001) / \\
(0.0001)\end{array}$ & $\begin{array}{l}191 \pm 8 / \\
(0.003)\end{array}$ & $\begin{array}{l}13 \pm 1 / \\
(0.03)\end{array}$ & $42 \pm 4(0.0001) /(0.0001)$ & $\begin{array}{l}152 \pm 7 / \\
(0.0001)\end{array}$ & a \\
\hline Biorepaire & $15 \pm 10(0.0001)$ & $64 \pm 54$ & $85 \pm 52$ & $3 \pm 1(0.0001)$ & $74 \pm 7$ & a \\
\hline
\end{tabular}

Abbreviations: CCDT, completely closed dentinal tubules; ESTP, eggshell toothpaste; ODT, opened dentinal tubules, PCDT, partially closed dentinal tubules; SD, standard deviation; TNPsESTP, titanium dioxide nanoparticles eggshell toothpaste.

Note: Both $\left({ }^{*}\right)$ and $(¥)$ represent the $t$-tailed $p$-value when negative (etched dentin) and positive control used, respectively.

${ }^{a}$ Difficult to count the number of dentinal tubules. 

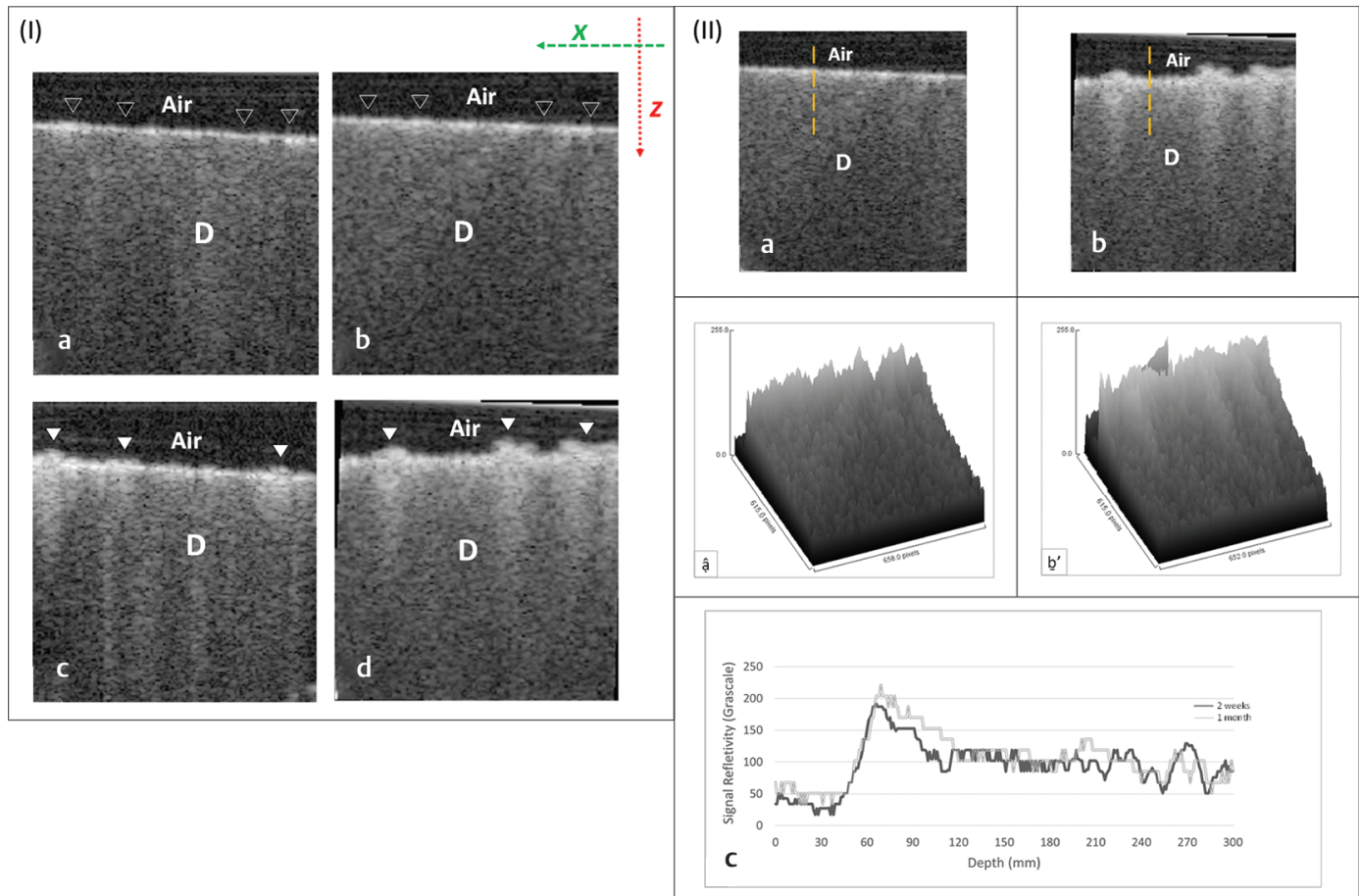

Fig. 3 TNPsESTP group: (I) Typical B-scans after 2 weeks (a, b) and 1 month (c, d). The signal reflectivity from the dentin surface along Z-axis increased after 1 month (solid white triangles) compared with 2 weeks (hollow triangles); this was also associated with a change in surface topography. (II) Representative OCT scans and their signals analyses after 2 weeks (a, ậ) and 1 month (b, b'); the vertical broken lines represent A-scan. As seen, an intense backscattered reflection was observed after 1 month (b) in comparison to the light bright band of pixels seen after 2 weeks (a). The presented surface plots (ậ, b'), corresponding to the OCT scans in (a) and (b), respectively, showed an increase in surface density as indicated by the difference in grayscale peaks. The signal reflectivity at the outer dentin surface after 1 month was greater than 2 weeks (c). D, dentin; OCT, optical coherence tomography; TNPsESTP, titanium dioxide nanoparticles eggshell toothpaste.
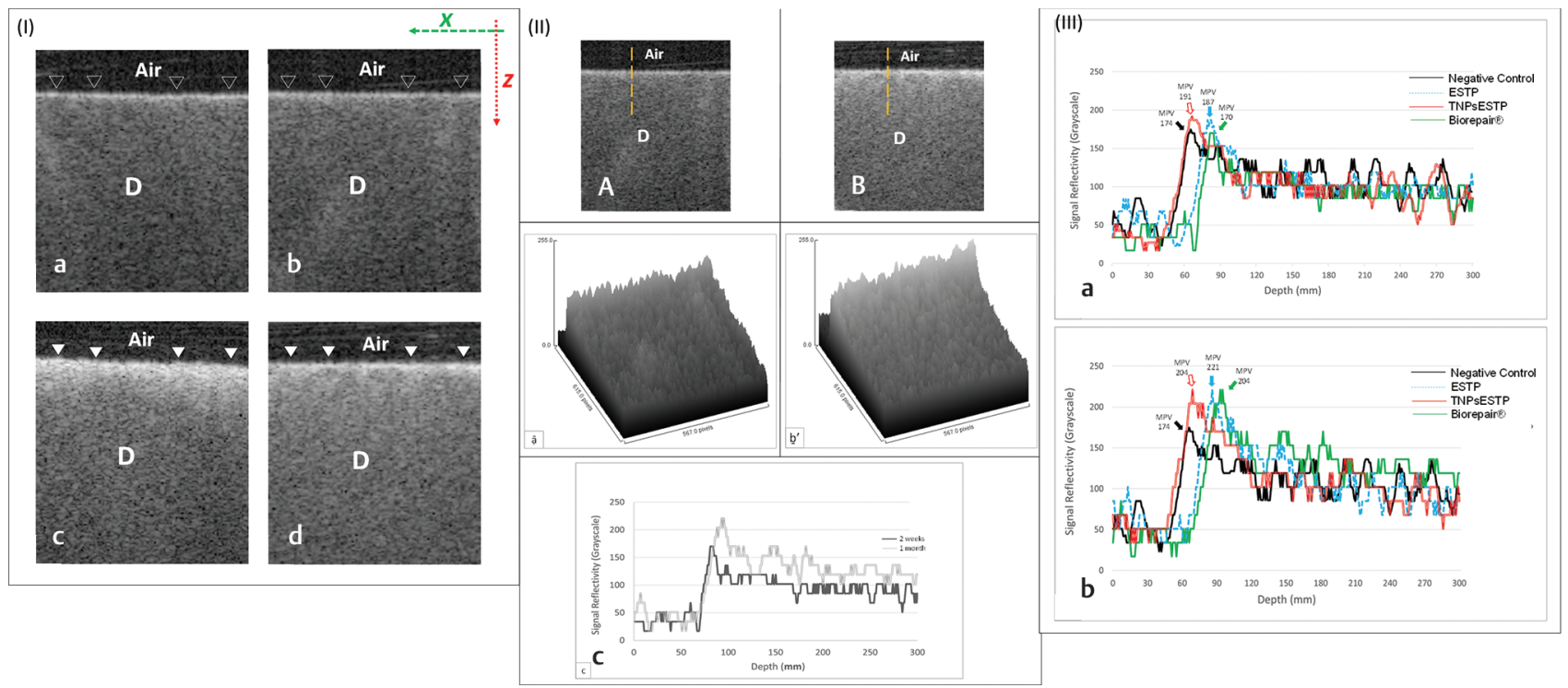

Fig. 4 Biorepair (positive control) group: (I) Representative B-scans for 2 weeks (a, b) and 1 month (c, d). A marked increase in surface reflectivity of dentin (solid white triangles) along Z-axis was observed in comparison to the same area indicated by hollow triangles. (II) Representative OCT scans and their signals analyses after 2 weeks (a, â) and 1 month (b, b'); the vertical broken lines represent A-scan. After 2 weeks, a light bright band of pixels was seen along the surface in comparison to the intense backscattered reflection after 1 month. The presented surface plots (ậ, b'), corresponding to the OCT scans in (a) and (b), respectively, showed an increase in surface density as indicated by the difference in grayscale peaks. The signal reflectivity at the outer dentin surface after 1 month was greater than 2 weeks (c). (III) Representative plot of signals for different groups after 2 weeks (a) and 1 month (b) acquired by A-scan measurement of MPV. D, dentin; MPV, maximum pixel value; $\mathrm{OCT}$, optical coherence tomography. 


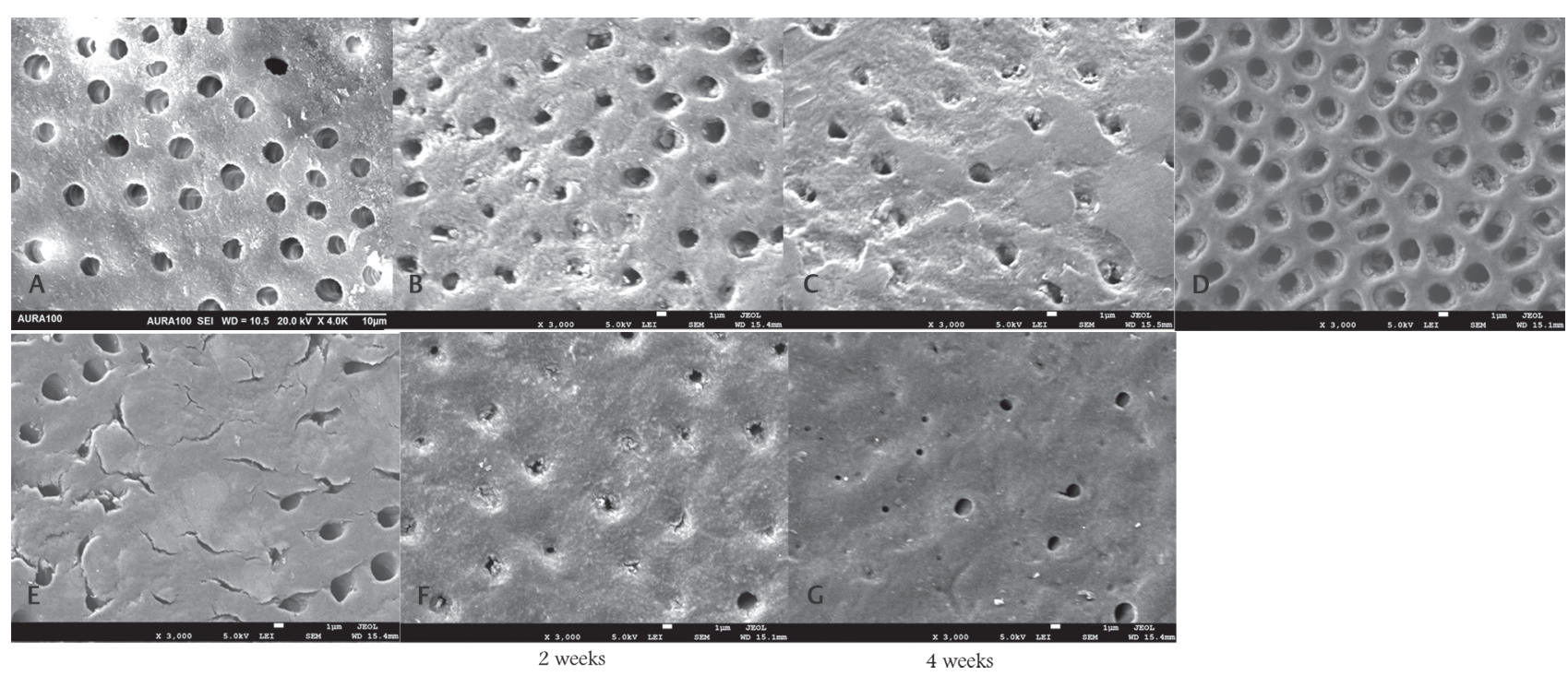

Fig. 5 Scanning electron microscopy images of etched dentin (A), etched dentin after treatment with ESTP (B, C), TNPsESTP (D, E), and Biorepair (F, G) for 2 weeks and 1 month. ESTP, eggshell toothpaste; TNPsESTP, titanium dioxide nanoparticles eggshell toothpaste.

Both ESTP and TNPsESTP showed significantly higher number of partially closed dentinal tubules at 4 weeks than Biorepair.

\section{Discussion}

This study investigated the remineralizing potential of the experimental eggshell-based toothpastes in comparison to etched dentin as a negative control and Biorepair as a positive control.

The CP-OCT system, based on near-infrared imaging, scans the substrates in a noninvasive mode. Changes in the mineral content of dental tissues is usually associated with some surface changes that will be perceived as light scattering on CP-OCT grayscale. Each pixel in a grayscale image has a solitary value conveying only intensity information. The gray shades on an image ranges from black (i.e., zero intensity) to white (i.e., maximum intensity of 255). Unlike swept-source OCT, CP-OCT contains a second polarizer that eliminates the "specular reflection" that forms over the imaged flat surfaces. CP-OCT had been therefore successfully used to investigate the changes in dental tissues. ${ }^{21,23}$ When the light is emitted from the OCT, it will pass through the air into dentin surface. As the refractive index (RI) of air is 1, the light passes through the air without any scattering or reflection. Due to the increase in RI of dentin (1.4-1.6), ${ }^{24}$ the speed of light will decrease when it reaches the dentin surface. Thus, on the OCT grayscale, the air appears as dark pixels, while any change in dentin surface appears as dark and bright pixels. Furthermore, with any increase in substrate density, the OCT light will undergo reflection and/or refraction. Therefore, the MPV was used to detect the changes in dentin surface. ${ }^{25-27}$

In this study, etching with $37 \%$ phosphoric acid for 60 seconds was used to simulate the hypersensitive dentin containing opened dentinal tubules. Toothpastes were applied to etched dentin using a toothbrush simulator with controlled movement and force application. The tooth brushing was carried out using classic soft brushes to simulate the clinical situation of DH where soft brushes are recommended. ${ }^{28}$ Circular motion was chosen for brushing as an effective way of removing dental plaque and maintaining gingival health. ${ }^{29}$ The number of brushing cycles was calculated assuming the ideal scenario for routine tooth brushing (three times/day and each time takes approximately 3 minutes) and the number of teeth is 28 . According to this assumption, each tooth is subjected to 20 seconds of brushing/day which is nearly double that used in other studies. ${ }^{30,31}$ This difference could be related to the difference in number and time of brushing per day. ${ }^{30,31}$ Finally, the brushing time was then converted into brushing cycles after considering the time taken for each brushing cycle under the used testing setting. The tooth brushing was carried out for 2 weeks and 1 month; the effect of acid or temperature challenge will be considered in future work. The efficacy of the experimental toothpastes was studied by their ability to block dentinal tubules and remineralize the intertubular dentin.

As observed the experimental toothpastes showed comparable results to Biorepair. This could be related to the release of calcium, phosphorus, and fluoride ions that are essential for remineralization. With TNPsESTP, the presence of TNPs could also have accounted for dentinal tubules' occlusion. The results of this study are in agreement with those obtained by Onwubu et $\mathrm{al}^{32-34}$ In one of Onwubu et al's studies, ${ }^{32}$ a slurry of both ESP with or without TNPs was used. The slurry was prepared by mixing $1 \mathrm{~g}$ of powder with $40 \mathrm{~mL}$ of deionized water. In the present study, however, other ingredients as shown earlier were included in the paste and the slurry was prepared at powder:liquid ratio of $1: 3$ to produce a paste with a consistency similar to the regular toothpaste. The samples in Onwubu et al's study were shaken for 3 hours in the presence of eggshell slurry, then they were subjected to $2 \mathrm{wt} \%$ citric acid challenge for 5 minutes. In the present study, however, brushing of etched dentin with the eggshell paste was attempted using the toothbrush simulator to mimic the clinical application of the proposed paste. 
The use of acid challenge however will be considered in the future work to check the stability of tubules occlusion due to paste applications. In another study by Onwubu et al,33 the slurry was prepared at a ratio of $100 \mathrm{mg}$ of ESP to $200 \mu \mathrm{L}$ of deionized water and tooth brushing was carried out using a battery-powered toothbrush twice/daily for 7days, 1 minute each time; the samples were kept in saliva in-between brushing. Regardless of this short time period, a significant reduction in number of opened dentinal tubules was observed with the eggshell paste when compared with the commercially available Sensodyne Repair \& Protect and Colgate Pro-Relief toothpastes. Furthermore, in Onwubu et al's studies, bovine incisors were used; however, in the present study, human extracted molar teeth were used. ${ }^{32-34}$

With Biorepair, the active ingredient, responsible for occlusion of dentinal tubule and dentin remineralization, is nanohydroxyapatite. The presence of hydroxyapatite in a nanoscale enhances its rapid dissolution and hence early occlusion of dentinal tubules. ${ }^{35}$ Furthermore, these nanocrystals are similar in morphology and structure to tooth apatite. $^{36}$ The released ions could act as a template for crystal growth and mineralization, and this could explain the increase in number of occluded dentinal tubules with time. According to the results of the present study, there was no strong evidence to reject the null hypothesis.

\section{Conclusion}

Under the limitation of the present study, the experimental toothpastes were effective in the occlusion of dentinal tubules and remineralizing of intertubular dentin. They also showed comparable results to Biorepair Fast Sensitive Repair. Therefore, they could be potentially used as a cost-effective treatment for $\mathrm{DH}$.

\section{Conflict of Interest}

None declared.

\section{References}

1 Liu X-X, Tenenbaum HC, Wilder RS, Quock R, Hewlett ER, Ren Y-F. Pathogenesis, diagnosis and management of dentin hypersensitivity: an evidence-based overview for dental practitioners. BMC Oral Health 2020;20(1):220

2 Arrais CAG, Chan DCN, Giannini M. Effects of desensitizing agents on dentinal tubule occlusion. J Appl Oral Sci 2004;12(2):144-148

3 da Cruz LPD, Hill RG, Chen X, Gillam DG. Dentine Tubule occlusion by novel bioactive glass-based toothpastes. Int J Dent 2018;2018:5701638. Doi:10.1155/2018/5701638

4 Miglani S, Aggarwal V, Ahuja B. Dentin hypersensitivity: recent trends in management. J Conserv Dent 2010;13(4):218-224

5 Mathew MG, Soni AJ, Khan MM, Kauser A, Charan VSS, Akula SK. Efficacy of remineralizing agents to occlude dentinal tubules in primary teeth subjected to dentin hypersensitivity in vitro: SEM study. J Family Med Prim Care 2020;9(1):354-358

6 Tschoppe P, Zandim DL, Martus P, Kielbassa AM. Enamel and dentine remineralization by nano-hydroxyapatite toothpastes. J Dent 2011;39(6):430-437

7 Kumar VL, Itthagarun A, King NM. The effect of casein phosphopeptide-amorphous calcium phosphate on remineralization of artificial caries-like lesions: an in vitro study. Aust Dent J 2008;53(1):34-40

8 Jena A, Kala S, Shashirekha G. Comparing the effectiveness of four desensitizing toothpastes on dentinal tubule occlusion: a scanning electron microscope analysis. J Conserv Dent 2017;20(4):269-272

9 Rovenský J, Stancíková M, Masaryk P, Svík K, Istok R. Eggshell calcium in the prevention and treatment of osteoporosis. Int J Clin Pharmacol Res 2003;23(2-3):83-92

10 Schaafsma A, van Doormaal JJ, Muskiet FA, Hofstede GJ, Pakan I, van der Veer E. Positive effects of a chicken eggshell powder-enriched vitamin-mineral supplement on femoral neck bone mineral density in healthy late post-menopausal Dutch women. Br J Nutr 2002;87(3):267-275

11 Identification of inorganic compounds in eggshell as a dental remineralization material. J Dentomaxillofacial Sci 2017;2(3):168-171

12 Haghgoo R, Mehran M, Ahmadvand M, Ahmadvand MJ. Remineralization effect of eggshell versus nano-hydroxyapatite on caries-like lesions in permanent teeth (in vitro). J Int Oral Health 2016;8(4):435-439 doi:10.2047/jioh-08-04-05

13 Schaafsma A, Pakan I. Short-term effects of a chicken egg shell powder enriched dairy-based products on bone mineral density in persons with osteoporosis or osteopenia. Bratisl Lek Listy 1999;100(12):651-656

14 Krompecher I. Antirachitic and anti-anemic effects of eggshell [in Hungarian]. Gyermekgyogyaszat 1959;10(2):42-50

15 Salah M, Kataia MM, Kataia EM. El Din EA, Essa ME. Evaluation of eggshell powder as an experimental direct pulp capping material. Future Dent J 2018;4(2):160-164

16 Mony B, Ebenezar AVR, Ghani MF, Narayanan A, S A, Mohan AG. Effect of chicken egg shell powder solution on early enamel carious lesions: an in vitro preliminary study. J Clin Diagn Res 2015;9(3):ZC30-ZC32

17 Buzala MAR, Kobayashi CAN. Sources of fluoride intake and risk of dental fluorosis. Actual Osteol 2007;3(1):13-24

18 Azizi-Lalabadi M, Ehsani A, Divband B, Alizadeh-Sani M. Antimicrobial activity of titanium dioxide and zinc oxide nanoparticles supported in $4 \mathrm{~A}$ zeolite and evaluation the morphological characteristic. Sci Rep 2019;9(1):17439

19 Park I-S. Anaesthetic efficacy and physiological response of clove oil and lidocaine- $\mathrm{HCl}$ on river puffer, Takifugu obscurus and tiger puffer, T. rubripes. Dev Reprod 2019;23(1):21-34

20 Marya CM, Satija G, J A, Nagpal R, Kapoor R, Ahmad A. In vitro inhibitory effect of clove essential oil and its two active principles on tooth decalcification by apple juice. Int J Dent 2012;2012:759618

21 Bakhsh TA, Al-Jeffery B, Zaatari G, et al. Evaluation of the adaptation of dental polymers using cross-polarization optical coherence tomography. Biomed Phys Eng Express 2019;5(2):025014

22 Turkistani A, Almutairi M, Banakhar N, et al. Optical evaluation of enamel microleakage with one-step self-etch adhesives. Photomed Laser Surg 2018;36(11):589-594

23 Chan $\mathrm{KH}$, Tom H, Lee RC, et al. Clinical monitoring of smooth surface enamel lesions using CP-OCT during nonsurgical intervention. Lasers Surg Med 2016;48(10):915-923

24 Hariri I, Sadr A, Nakashima S, Shimada Y, Tagami J, Sumi Y. Estimation of the enamel and dentin mineral content from the refractive index. Caries Res 2013;47(1):18-26

25 Lenton P, Rudney J, Fok A, Jones RS. Clinical cross-polarization optical coherence tomography assessment of subsurface enamel below dental resin composite restorations. J Med Imaging (Bellingham) 2014;1(1):016001

26 Nee A, Chan K, Kang H, Staninec M, Darling CL, Fried D. Longitudinal monitoring of demineralization peripheral to orthodontic brackets using cross polarization optical coherence tomography. J Dent 2014;42(5):547-555 
27 Rasmussen K, Reilly C, Li Y, Jones RS. Real-time imaging of anti-biofilm effects using CP-OCT. Biotechnol Bioeng 2016;113(1):198-205

28 SehmiH,OlleyRC. The effect of toothbrushabrasionforceondentine hypersensitivity in-vitro. J Dent 2015;43(12):1442-1447

29 Hapsari I, Hunsrisakhun J. Comparison of modified circular and natural toothbrushing methods in effectiveness of dental plaque removal and gingival improvement. J Int Oral Health 2020;12(1):20-26

30 Creeth JE, Gallagher A, Sowinski J, et al. The effect of brushing time and dentifrice on dental plaque removal in vivo. J Dent Hyg 2009;83(3):111-116

31 Bizhang M, Schmidt I, Chun YP, Arnold WH, Zimmer S. Toothbrush abrasivity in a long-term simulation on human dentin depends on brushing mode and bristle arrangement. PLoS One 2017;12(2):e0172060

32 Onwubu SC, Mdluli PS, Singh S, Tlapana T. A novel application of nano eggshell/titanium dioxide composite on occluding dentine tubules: an in vitro study. Braz Oral Res 2019;33(e16) :e016

33 Onwubu SC, Mdluli PS, Singh S, Bharuth V, Makgobole MU. Evaluation of the occluding characteristics of nanosized eggshell/titanium dioxide with or without saliva. Eur J Dent 2019;13(4):547-555

34 Onwubu SC, Mdluli PS, Singh S, Bharuth V. Remineralization potential of a modified eggshell-titanium composite-scanning electron microscope study. Eur J Dent 2019;13(2):187-192

35 Bologa E, Stoleriu S, Iovan G, Ghiorghe CA, Nica I, Andrian S, Amza OE. Effects of dentifrices containing nanohydroxyapatite on dentinal tubule occlusion-a scanning electron microscopy and EDX study. Appl Sci (Basel) 2020;10:6513

36 Scribante A, Dermenaki Farahani MR, Marino G, et al. Biomimetic effect of nano-hydroxyapatite in demineralized enamel before orthodontic bonding of brackets and attachments: visual, adhesion strength, and hardness in in vitro tests. BioMed Res Int 2020;2020:6747498 